\title{
Der Beitrag der Bundesrepublik Deutschland zum IGCP Project No. 61 „Sea Level Movements during the Last Deglacial Hemicycle (ca. 15.000 years)"
}

\author{
Hansjörg Streif \& Renier Vinken *) \\ International Geological Correlation Holocene Project, sea level, Upper Pleistocene, \\ Federal Republic of Germany, North Sea, Baltic Sea
}

$\mathrm{Kurzf}$ assung: In einem Uberblick werden die Forschungsziele, die Organisation sowie bisherige Aktivitäten und zukünftige Vorhaben im Rahmen des IGCP Project No. 61 - Sea Level Project - dargestellt.

Spezieller werden die nationalen Beiträge von Seiten der Bundesrepublik Deutschland behandelt, die durch die Deutsche Forschungsgemeinschaft gefördert werden. Diese umfassen regionale geologische Untersuchungen sowie interdisziplinäre Studien über die Bestimmung früherer Meeresspiegelstände und deren Verlagerungstendenzen im südlichen Nord- und Ostseegebiet. Zusätzlich liefert die Entwicklung von Computer-Programmen zur EDV-Auswertung von Seespiegeldaten einen überregionalen Beitrag zum internationalen Sea Level Project. [The Contribution of the Federal Republic of Germany to IGCP Project No. 61 Sea Level]
Movements during the Last Deglacial Hemicycle (ca. 15000 years)]

A bstract: The scientific aims, the organization, and present activities, as well as plans for the future of the IGCP Project No. 61 - Sea Level Project -, are presented in an overview.

The national contribution of the Federal Republic of Germany, which is sponsored by the Deutsche Forschungsgemeinschaft, is especially emphasised. The contribution comprises regional geological field work, as well as interdisciplinary studies on the determination of previous sea levels and tendencies of sea level changes in the southern North Sea and Baltic Sea region. Additionally the development of computer programmes for evaluation of sea level data provides a supraregional contribution to the international Sea Level Project.

\section{Einleitung}

Das Forschungsvorhaben „Sea Level Movements during the Last Deglacial Hemicycle (ca. 15.000 years)" geht auf die Initiative von Prof. Dr. Thiadens, dem früheren Direktor des Rijks Geologische Dienst der Niederlande, zurück. Das Vorhaben wurde vom Board des „International Geological Correlation Programme (IGCP)“ anläßlich seiner zweiten Sitzung im April 1974 in Wien als IGCP-Project No. 61 (Kurzbezeichnung „Sea Level Project") angenommen.

Der Ansatz zu diesem Sea Level Project hatte sich aus zwei Gesichtspunkten ergeben. - Die Flachküstenregionen der Erde sind von größter wirtschaftlicher Bedeutung, aber gelten aus geologischer Sicht als gefährdet. Sie gehören einerseits zu den dicht besiedelten Regionen der Erde und bieten günstige Voraussetzungen für die Landwirtschaft. Seit alters her haben sich vor allem im Bereich der Flußmündungen Handelszentren entwickelt, und in zunehmendem Maße entstehen hier Industrieballungsgebiete. Andererseits hat sich der Meeresspiegel - überwiegend durch klimatisch gesteuerte Verschiebungen in der EisWasser-Bilanz der Erde - erheblich verschoben, wobei die Geschwindigkeiten dieser Verschiebungen z. T. weit über das gegenwärtige Ausmaß hinausgingen. Anscheinend hat sich zur Zeit ein relativ stabiler Gleichgewichtszustand eingependelt, der jedoch von begrenzter Dauer sein dürfte und sehr sensibel auf veränderte Umweltbedingungen reagieren kann.

*) Anschrift der Verfasser: Dr. H. S t r e if u. Dr. R. Vin k e n, Niedersächsisches Landesamt für Bodenforschung, Stilleweg 2, D-3000 Hannover 51. 
Hauptziel des Projekts ist es, eine Kurve vom Trend der Verschiebung des mittleren Meeresspiegels seit dem Höhepunkt der letzten Vereisung bis heute zu entwickeln. Diese Kurve soll die klimatisch bedingten Veränderungen des hydrologischen Gleichgewichts zwischen Eis und Wasser wiedergeben. Dabei werden Einzelbeobachtungen über die relativen Seespiegelverschiebungen aus allen Gebieten der Erde betrachtet, Rückschlüsse auf lokale Krustenbewegungen entlang Küsten gezogen und Basisdaten über die Festigkeit und Elastizität der Erdkruste gewonnen.

\section{Internationale Aktivitäten}

Anläßlich eines ersten Treffens am 26. und 27. 9. 1974 formierte sich eine vorläufige Arbeitsgruppe für das IGCP Project No. 61, und es wurde ein Grundkonzept der Arbeitsschwerpunkte und der Arbeitsteilung entworfen. Dieses wurde auf den späteren Jahresversammlungen fortentwickelt und ergänzt. Weitere Tagungsorte waren:

1975: 22.-23. 12. Haarlem, Niederlande

1976: 5.-12. 12. Dakar, Senegal (organisiert durch die Association Sénégalaise pour l'Etude du Quaternaire, ASEQUA)

1977: 16. 8. Birmingham, England (im Zusammenhang mit dem X. Internationalen Kongreß der INQUA)

1978: 11.-18. 9. Sao Paulo, Brasilien (im Rahmen des „1978 International Symposium on Coastal Evolution in the Quaternary")

1979: 20. 9. Texel, Niederlande (im Rahmen des „International Meeting on Holocene Marine Sedimentation in the North Sea Basin“).

Die weitere Planung sieht vor, Jahrestreffen 1980 anläßlich des Internationalen Geologenkongresses in Paris und 1981 in South Carolina, U.S.A., abzuhalten. Es ist beabsichtigt, das IGCP Project No. 61 zum INQUA-Kongreß in Moskau 1982 abzuschließen.

Die Administration des Projektes setzt sich wie folgt zusammen. Zum internationalen Projektleiter wurde Prof. Dr. A. L. Bloom, Department of Geological Sciences, Cornell University, Ithaca New York, U.S.A., gewählt. Dieser präsidiert einem Executive Board (Mitglieder vgl. Anhang 1). Offiziell beteiligen sich 26 Länder am IGCP Project No. 61. Es sind dies: Australien, Belgien, Benin, Brasilien, Bundesrepublik Deutschland, Canada, Cypern, Dänemark, Finnland, Frankreich, Großbritannien, Irland, Island, Israel, Italien, Japan, Monako, Niederlande, Neuseeland, Norwegen, Schweden, Spanien, Südkorea, Türkei, Union der Sozialistischen Sowjetrepubliken und Vereinigte Staaten von Amerika. Ene weitere Beteiligung ist geplant oder eine enge inoffizielle Verbindung besteht zu Forschergruppen in Argentinien, Bulgarien, Indien, Indonesien, Malaysia, Mexiko, Nigeria, Philippinen, Senegal, Südafrika, Volksrepublik China und zur Volksrepublik Kongo.

Die Mehrzahl der am Sea Level Project beteiligten Wissenschaftler ist gleichzeitig auch in INQUA-Kommissionen tätig, wodurch sich eine befruchtende Wechselwirkung ergibt. Einerseits gelangen weltweit gestreut sehr zuverlässige Befunde über die relativen Meeresspiegelverschiebungen in das Sea Level Project. Andererseits wirkt dieses wieder in die regionalen Subkommissionen der INQUA hinein, insbesondere in die „Subcommission for Shorelines“, „for Holocene Stratigraphy“ und "for Neotectonics“. Enge Verbindungen bestehen darüber hinaus auch zu anderen internationalen wissenschaftlichen Programmen, die in einer Beziehung zu Meeresspiegelschwankungen stehen, so z. B. zum Geodynamik Programm und zum SCAR-Programm, das sich u. a. mit den Volumenänderungen des antarktischen Eisschildes befaßt.

Als turnusmäßige Mitteilungen über die Forschungsaktivitäten zum Sea Level Project und zur Küstenentwicklung im Quartär erscheinen auf internationaler Ebene folgende Periodika: 
- NIVMER Information; Herausgeber: Comité National Français du P.I.C.G. (= I.G.C.P.). Vorsitzender: Prof. H. Faure, Lab. de Géologie du Quaternaire, Centre Universitaire de Marseille-Luminy, F 13288 Marseille-Cedex 2

Secrétaire Général: Dr. P. Pirazzoli, Laboratoire de Géographie Ecole Normale Supérieure, 1 rue Maurice Arnoux, F-92120 Montrouge

- Sea-Level; Information Bulletin of I.G.C.P. Project No. 61. Internat. Project-Leader: Prof. Dr. A. L. Bloom, Dep. of Geolog. Sciences, Cornell University, Ithaca New York 14853, U.S.A.

Compiler: Dr. M. J. Toolex, Dep. of Geography, The University of Durham, Science Laboratories, Druham, DH1 3LE, England.

- Litoralia; Herausgeber: INQUA Commission on Quaternary Shorelines. - Präsident: Prof. Dr. J. Colquhoun, Dep. of Geology, University of South Carolina, Columbia, S. C. 29208 , U.S.A.

Sekretär: Dr. D. Grant, Geol. Survey of Canada, 601 Booth Street, Ottawa, Ontario, Canada, K1A OE8.

Offizielle Kontakte zwischen den in der Bundesrepublik tätigen Küstenforschern und den o.g. internationalen Organisationen bestehen über eine Reihe von Personen, die in Anhang 2 aufgeführt sind.

Im Zuge internationaler Zusammenarbeit wurden bisher folgende Ziele erreicht. Ein Computer Programm für die Erfassung, Verarbeitung und Wiedergabe der globalen Seespiegeldaten wurde entwickelt (vgl. Preuss 1980 dieser Band). In einem Atlas wurde versucht, sämtliche vorhandenen Seespiegel-Schwankungskurven zu sammeln, um eine Grundlage für überregionale Vergleiche zu gewinnen (BLоom 1977). Es ist beabsichtigt, diese Sammlung laufend zu ergänzen. Einen weiteren Schwerpunkt bilden Modellberechnungen für die eis- und hydroisostatischen Komponenten der Meeresspiegelschwankungen (Clark et al. 1978; Clark \& Bloom 1979). Ein Handbuch für die Sammlung und Auswertung von Seespiegeldaten befindet sich in Arbeit, dessen Entwurf von VAN DE PLASSCHE (1977) vorgelegt wurde und das in stark erweiterter Form 1981 erscheinen soll. Unverkennbar ist auch die stimulierende Wirkung, die das Sea Level Project auf detaillierte regionale Untersuchungen weltweit gehabt hat.

\section{Nationale Aktivitäten in der Bundesrepublik Deutschland}

Die im Rahmen des IGCP Projectes No. 61 durchgeführten Arbeiten in der Bundesrepublik Deutschland wurden von Seiten der Deutschen Forschungsgemeinschaft gefördert. Koordiniert durch den nationalen Projektleiter R. Vinken befaßten sich zwei spezielle Forschungsprojekte mit dem Problem der Seespiegelverschiebungen mit den regionalen Schwerpunkten im Raum Eiderstedt, Schleswig-Holstein, und dem Gebiet zwischen Emsund Wesermündung. Mit dieser Auswahl der Untersuchungsgebiete wird die Übergangszone zwischen dem Senkungsgebiet der Niederlande und dem isostatischen Hebungsgebiet Jütlands geschlossen.

Die von der Arbeitsgruppe Elwert \& Schneider (GLA Schleswig-Holstein) durchgeführten Arbeiten wurden 1975 aufgenommen und 1979 abgeschlossen. Eine zusammenfassende Veröffentlichung der Befunde (ELwERT \& SCHNeIder 1979) ist in Vorbereitung. Den Schwerpunkt dieser Arbeit bilden paläogeographische Karten für verschiedene Tiefenbereiche und Entwicklungszustände Eiderstedts. Faziesanalysen, basierend auf Schilluntersuchungen und geobotanischen Studien, stratigraphische Gliederungen mit Hilfe von palynologischen Methoden und ${ }^{14} \mathrm{C}$-Altersbestimmungen ergänzen diese Befunde und erlauben Rückschlüsse über die Dynamik des Meeresspiegel-Anstiegsgeschehens.

Die von der Arbeitsgruppe Barckhausen, Preuss, Streif, Tabat \& Vinken (NLfB Hannover) durchgeführten Arbeiten sind ebenfalls 1975 angelaufen, ihr Abschluß ist für 
1980 geplant. Die feldgeologischen Arbeiten in Niedersachsen hatten ihren Schwerpunkt im Bereich der Wesermündung, wobei speziell die holozäne Entwicklung des östlichen Weserufers zwischen Neuenkirchen im Süden und Arensch im Norden untersucht worden ist (Preuss 1979). Weitere Arbeiten wurden im Bereich der unteren Ems, im Gebiet des Jadebusens und an der unteren Elbe durchgeführt. - Wesentlichen Arbeitsanteil machten auch die Entwicklung des Formblattes und der EDV-Programme zur Dokumentation, Verarbeitung und Auswertung von Seespiegeldaten aus (vgl. Preuss dieser Band). Hier wurde von deutscher Seite ein weit über den regionalen Bereich hinausgreifender Beitrag zum internationalen Sea Level Project geleistet.

Neben den genannten Untersuchungsprogrammen, die im Rahmen des IGCP Projects No. 61 von der Deutschen Forschungsgemeinschaft gefördert worden sind, laufen in der Bundesrepublik Deutschland zahlreiche andere Arbeiten unterschiedlichster Fachrichtungen, die in sehr enger Beziehung zum Sea Level Project stehen. Um daher das gesamte Spektrum interdisziplinärer Beiträge zum Thema Meeresspiegelschwankungen zu erfassen, wurden auf Anregung der Deutschen Forschungsgemeinschaft bislang 2 DFG-Rundgespräche „Holocene Sea Level Changes“ in Hannover durchgeführt (26.-27. Oktober 1976 und 1.-2. März 1979). Daran waren neben Geologen und Paläobotanikern vor allem Archäologen, Geographen, Hydrologen, Sedimentologen sowie Wasserbauer beteiligt.

Bei den beiden DFG-Rundgesprächen standen folgende Themenkreise im Vordergrund der Betrachtungen:

- Austausch über neue Ergebnisse und laufende Arbeiten

- Festlegung von Kriterien für eine einheitliche Sedimentbeschreibung und für die genetische Zuordnung der Sedimente

- Aussagewert und -genauigkeit von lithologischen, biologischen und anthropogenen Meeresspiegelindikatoren

- Erstellung eines Kriterienkataloges für den Nachweis von Regressionen

- Vergleich, Verknüpfung und Brauchbarkeit stratigraphischer Systeme

- EDV-Auswertung von Seespiegeldaten und Computermodelle.

Die erörterten Themen sind z. T. Gegenstand der Veröffentlichungen dieses Bandes (Behre \& Streif, Brandt, Figge, Hanisch, Hoffmann, Klug, Pirazzoli et al., van de Plassche, Preuss und Wunderlich), z. T. sind sie an anderer Stelle veröffentlicht worden.

Hervorzuheben sind hier vor allem die regionalen landschafts- und siedlungsgeschichtlichen Arbeiten im Bereich der Westküste von Schleswig-Holstein (HigelKE et al. 1979; Averdiek 1981; Harck 1981; Hoffmann 1981). Anhand von Untersuchungen im Küstenbereich südlich Cuxhaven entwickelte LiNKe (1979) neue Hypothesen über die Auswirkungen sturmflutfreier und sturmflutaktiver Phasen auf die Höhenlage von Seespiegelanzeigern und über ihre Bedeutung für das Meeresspiegel-Anstiegsgeschehen. Regional ergänzende Befunde über das Küstenholozän am Ostufer der Wesermündung veröffentlichte Preuss (1979). Aus Einzelbeobachtungen über die Höhen früherer Sturmflutwasserstände und langzeitigen Beobachtungen von Pegelständen ermittelte RoHDE $(1975,1977)$ die Beträge des jüngsten Seespiegelanstiegs an der deutschen Nordseeküste.

Neuere kompilatorische Arbeiten über die Entwicklung des Küstenraumes und des deutschen Nordseesektors sind von Behre (1978), Streif \& Köster (1978), Behre, Menke \& Streif (1979) und Ludwig, Müller \& STReif (1979) veröffentlicht worden. Der von Oele, Schüttenhelm \& Wiggers (1979) editierte Band „The Quaternary History of the North Sea" enthält darüber hinausgehend zahlreiche Beiträge aus allen Nordsee-Anrainerstaaten. 


\section{Schlußbetrachtungen}

Im ursprünglichen Ansatz ging das IGCP Project No. 61 davon aus, daß es nach Abschluß der Arbeiten möglich sein sollte, die eustatische Komponente des Meeresspiegel-Anstiegsgeschehens in Gestalt einer Kurve mit hoher Genauigkeit zu erfassen. Die Summe der übrigen Komponenten hätte dann durch einen einfachen Vergleich der Kurven des relativen lokalen Meeresspiegelanstiegs mit der des eustatischen globalen Anstiegs ermittelt werden können.

Die kritische Sichtung der Basisdaten und neuere Erkenntnisse führten dazu, daß diese Erwartungen etwas zurückgenommen wurden. - Die Fehlerintervalle der ${ }^{14} \mathrm{C}$-Alter und die begrenzten Aussagegenauigkeiten anderer Datierungsmethoden, die Auswirkungen unterschiedlicher Sedimentationsraten und Setzungsbeträge und das Problem, eine bestimmte Probenentnahmehöhe auf einen früheren Seespiegelstand $\mathrm{zu}$ beziehen, machen deutlich, daß man bislang nicht in der Lage ist, Seespiegel-Anstiegskurven zu zeichnen, deren Genauigkeit im Dezimeter-Bereich liegt, was viele veröffentlichte Kurven suggerieren. Bei der Berücksichtigung allein dieser Unsicherheiten können allenfalls Höhenintervalle eingegrenzt werden, innerhalb derer frühere Meeresspiegelstände gelegen haben müssen. Folglich läßt sich keine präzise Meeresspiegel-Anstiegskurve konstruieren, sondern nur ein mehr oder weniger breites Band, wobei Meeresspiegelschwankungen, die innerhalb dieser Bandbreite liegen, methodisch bedingt maskiert bleiben.

Von den das Meeresspiegel-Anstiegsgeschehen steuernden geophysikalischen Faktoren - glazialeustatische Meeresspiegelschwankungen, isostatische Ausgleichsbewegungen, epirogenetische Bewegungen und geoidale Effekte - wurden im Verlauf der internationalen Arbeiten insbesondere die Probleme der Isostasie und der geoidalen Veränderungen behandelt.

Dem Problem der eis- und hydroisostatischen Komponenten hat man sich mit Hilfe von Computermodellen genähert (Farrell \& Clark 1976; Clark, Farrel \& Peletier 1978; Clark \& Bloom 1979). Diese Modelle gehen von einem elastischen bzw. viskosen Verhalten der Erde aus und von dem Konzept, daß das Abtauen der Eisschilde etwa 5000 v.h. abgeschlossen war. Durch die regionale Eisentlastung im Verbreitungsgebiet der Eisschilde und die damit einhergehende Belastung der gesamten Ozeanböden und tieferen Schelfgebiete mit zusätzlich ca. $100 \mathrm{~m}$ Wassersäule ist es zu einer uneinheitlichen Deformation der Erdkruste gekommen. Aus den Modellberechnungen ergibt sich eine Einteilung der Ozeane in 6 Zonen, die jeweils durch eine spezifische Form der Meeresspiegel-Anstiegskurve gekennzeichnet sind. Für 4 dieser Zonen werden Meeresspiegelstände vorausgesagt, die höher liegen als heute. Dies gilt auch für Bereiche, die weit außerhalb der eisisostatischen Hebungsgebiete liegen. Insgesamt kann die hydroisostatische Komponente der Meeresspiegelschwankungen bis ca. $10 \mathrm{~m}$ betragen und damit in vielen Gebieten den Hauptteil der mittel- und jungholozänen Meeresspiegeländerungen ausmachen. Vergleiche tatsächlich beobachteter Meeresspiegelstände und berechneter Höhen zeigen zufriedenstellende Übereinstimmung.

Die Suche nach einer global gültigen eustatischen Kurve wurde durch die kritischen Arbeiten Mörners (1976, 1977) über Verformungen des Geoids spürbar gebremst. Das Geoid ist eine Äquipotentialfläche des Erd-Schwerefeldes, die sich durch die Erdanziehung und -rotation ergibt, wobei die Geoidfläche im Bereich der Ozeane als geodätischer Seespiegel aufgefaßt wird. Unter der Annahme, daß die Form des Geoids jeweils durch die Tiefenlage der Grenzflächen Erdkruste/Erdmantel und Erdmantel/Erdkern bedingt ist, gelangt Mörner zu der Auffassung, daß sich das Geoid innerhalb der Erdgeschichte verformt haben muß. Solche Verformungen sollen auch innerhalb der relativ kurzen Zeit- 
spanne des Spätglazials und Holozäns aufgetreten sein, wobei Mörner bislang noch keine Abschätzung über das mögliche Gesamtausmaß der zu erwartenden Beträge gibt.

Eine vorläufige Bilanz aufgrund des heutigen Standes im IGCP Project No. 61 ergibt, daß neben den vielfältigen bekannten Komponenten eine Reihe bislang unberücksichtigter oder nicht quantifizierbarer Komponenten maßgeblichen Einfluß auf das MeeresspiegelAnstiegsgeschehen haben und sich auf die Fehlerintervalle, mit denen Seespiegeldaten behaftet sind, auswirken. Dennoch zeigt sich, daß charakteristische Typen von MeeresspiegelAnstiegskurven mit einer gewissen Gesetzmäßigkeit verteilt vorkommen, die in benachbarten Regionen zwar unterschiedlich aussehen können, aber unverkennbar Züge einer gegenseitigen Abhängigkeit aufweisen. Ein globaler systematischer Vergleich der Seespiegelbasisdaten mittels Computer sowie ein analytischer Vergleich der abgeleiteten Kurventypen ist für die verbleibenden Arbeitsjahre vorgesehen.

Der internationale und fachübergreifende Austausch im Rahmen des IGCP Projects No. 61 hat gemeinsam mit den durch die Deutsche Forschungsgemeinschaft geförderten Arbeiten im deutschen Küstenbereich sehr stimulierende Auswirkungen gehabt. Dies wird aus der Fülle in jüngster Zeit erschienener Veröffentlichungen und der Arbeiten im vorliegenden Band deutlich. Selbst in dem gut untersuchten deutschen Küstenbereich konnten zahlreiche neue Ergebnisse gewonnen, bestehende Informationslücken geschlossen und die Qualität neuer Meeresspiegeldaten verbessert werden. In weit höherem Maße gilt dies für die Erforschung des spätglazialen und holozänen Meeresspiegel-Anstiegsgeschehens in den Küstenzonen von Entwicklungsländern.

\section{Schriftenverzeichnis}

Averdiek, F.-R. (1981): Geobotanik des Sylter Holozäns. - Römisch-Germanische Forsch., 39: 147-172, Abb. 65-71, Beil. 10-22; Mainz. - [Im Druck.]

Benre, K.-E. (1978): Die Geschichte des Jadebusens und der Jade. - In: Reineck (Hrsg.): Das Watt - Ablagerungs- und Lebensraum: 39-49, Abb. 15-19; Frankfurt a. M.

-, Menke, B. \& Streif, H. (1979): The Quaternary geological development of the German part of the North Sea. - In: Oele, E. \& Schüttenhelm, R. T. E. \& Wiggers, A. J. (eds.) The Quaternary History of the North Sea. - Acta Univ. Ups. Symp. Univ. Ups. Annum Quingentesimum Celebrantis, 2: 85-113, 9 Abb.; Uppsala.

- \& Streif, H. (1980): Kriterien zu Meeresspiegel- und darauf bezogene Grundwasserabsenkungen. - Eiszeitalter u. Gegenwart, 30: 153-160; Hannover.

Bloom, A. L. (1977): Atlas of Sea-level curves. - Internat. Geol. Correlation Programme 61, Sea-Level Project. Dept. of Geol. Sci., Cornell University; Ithaca, N.Y.

Brandt, K. (1980): Die Höhenlage ur- und frühgeschichtlicher Wohnniveaus in nordwestdeutschen Marschengebieten als Höhenmarken ehemaliger Wasserstände. - Eiszeitalter u. Gegenwart, 30: 161-170, 2 Abb.; Hannover.

Clark, I. A. \& Bloom, A. L. (1979): Hydro-isostaty and Holocene emergence of South America. - Proc. "1978 Internat. Symp. coastal evolution Quatern.": 41-60, 9 Abb.; Sao Paulo.

- , Farrell, W. E. \& Peletier, W. R. (1978): Global changes in postglacial sea level: a numerical calculation. - Quat. Research, 9: 265-287, 23 Abb.; Washington.

Elwert, D. \& Schneider, W. M. (1979): Meeresspiegel-Schwankungen der Nordsee im Jungpleistozän und Holozän - Sea Level Project - Abschlußbericht 1979: 56 S., 2 Abb., 14 Anl.; unveröff. Ber. GLA Kiel.

FigGe, K. (1980): Das Elbe-Urstromtal im Bereich der Deutschen Bucht. - Eiszeitalter u. Gegenwart, 30: 203-211, 6 Abb.; Hannover.

Hanisch, J. (1980): Neue Meeresspiegeldaten aus dem Raum Wangerooge. - Eiszeitalter u. Gegenwart, 30: 221-228, 5 Abb.; Hannover.

HARCK, O. (1981): Landschaftsgeschichte und Archäologie an der Westküste der jütischen Halbinsel. - Römisch-Germanische Forsch., 39; 32-63, Abb. 22-34, Beil. 1; Mainz. - [Im Druck.] 
Higelke, B., Hoffmann, D., Kühn, H. J. \& MülleR-Wille, M. (1979): Geowissenschaftlich-archäologische Untersuchungen zur Landschafts- und Siedlungsgeschichte von Nordfriesland. Archäol. Korrespondenzblatt, 9, 2: 223-239,8 Abb., Taf. 32-34; Mainz.

Hoffmann, D. (1980): Meeresspiegeldaten aus landschafts- und siedlungsgeschichtlichen Untersuchungen auf Pellworm. - Eiszeitalter u. Gegenwart, 30: 229-236, 3 Abb.; Hannover.

- (1981): Küstenholozän zwischen Sylt und Föhr. - Römisch-Germanische Forsch., 39: 85130, Abb. 44-59, Beil. 2-9; Mainz. - [Im Druck.]

KLug, H. (1980): Der Anstieg des Ostseespiegels im deutschen Küstenraum seit dem Mittelatlantikum. - Eiszeitalter u. Gegenwart, 30: 237-252, 5 Abb., 1 Tab.; Hannover.

LiNKE, G. (1979): Ergebnisse geologischer Untersuchungen im Küstenbereich südlich Cuxhaven Ein Beitrag zur Diskussion holozäner Fragen. - Probl. Küstenforsch., 13: 39-83, 18 Abb.; Hildesheim.

Ludwig, G., MüLler, H. \& STREIF, H. (1979): Neuere Daten zum holozänen Meeresspiegelanstieg im Bereich der Deutschen Bucht. - Geol. Jb., D 32: 3-22, 2 Abb., 7 Tab., Hannover.

Mörner, N. A. (1976): Eustasy and geoid changes. - J. Geol., 84, 2: 123-151, 17 Abb., 1 Tab.; Chicago.

- (1977): Eustasy and instability of the geoid configuration. - GFF, 99 (4): 369-376, 7 Abb.; Stockholm.

Oele, E., Schüttenhelm, R. T. E. \& Wiggers, A. J. (Hrsg.) (1979): The Quaternary history of the North Sea. - Acta Univ. Ups. Symp. Univ. Ups. Annum Quingentesimum Celebrantis, 2: 248 S., Abb., Kt.; Uppsala.

Pirazzoli, P. A., Planchais, N., Rosset Moulinier, M. \& Thommeret, J. (1980): Paleogeographic Interpretation of a Peat Layer at Torson di Sotto (Lagoon of Venice, Italy). - Eiszeitalter u. Gegenwart, 30: 253-259, 3 fig.; Hannover.

Plassche, O. van DE (1977): A manual for sample collection and evaluation of sea level data (draft, unfinished). - 55 S., Abb., Amsterdam (Inst. Earth Sci., Freie Universität).

- (1980): Compaction and Other Sources of Error in Obtaining Sea-Level Data: Some Results and Consequences. - Eiszeitalter u. Gegenwart, 30: 171-181, 8 fig.; Hannover.

Preuss, H. (1979): Die holozäne Entwicklung der Nordseeküste im Gebiet der östlichen Wesermarsch. - Geol. Jb., A 53: 3-85, 25 Abb., 5 Tab.; Hannover.

- (1980): Computerauswertung von Seespiegeldaten. - Eiszeitalter u. Gegenwart, 30: 183201, 11 Abb.; Hannover.

RoHde, H. (1975): Wasserstandsbeobachtungen im Bereich der deutschen Nordseeküste vor der Mitte des 19. Jahrhunderts. - Die Küste, 28: 1-96, 26 Abb., 2 Tab.; Heide i. Holst.

- (1977): Sturmfluthöhen und säkularer Wasserstandsanstieg an der deutschen Nordseeküste. Die Küste, 30: 52-143, 17 Abb., 5 Tab.; Heide i. Holst.

Streif, H. \& Köster, R. (1978): Zur Geologie der deutschen Nordseeküste. - The geology of the German North Sea Coast. — Die Küste, 32: 30—49, 7 Abb.; Heide i. Holst.

Wunderlich, F. (1980): Transgression und Umlagerung im Gebiet des Helgoland-Riffs. - Eiszeitalter u. Gegenwart, 30: 213-220, 5 Abb.; Hannover.

Manuskript eingegangen am 15. 2. 1980. 


\section{An lage 1}

Mitglieder des Executive Board, internationale Arbeitsgruppe des IGCP Projects No. 61
A. L. BLoom
(Internationaler Projektleiter)
Department of Geological Sciences
Cornell University
Ithaca, New York, U.S.A.

H. FAURE

Laboratoire de Géologie du Quaternaire

Centre Universitaire de Marseille-Luminy/Frankreich

B. P. Hageman

Rijks Geologische Dienst

Haarlem/Niederlande

P. A. KAPLIN

Geographische Fakultät, Moskau

Staatsuniversität Moskau/UdSSR

K. Suguio

Departamento de Paleontologia e Estratigrafia Instituto de Geosciencas, Universidade de Sao Paulo Sao Paulo/Brasilien

M. J. Toolex Department of Geography, The University of Durham Durham/Großbritannien

R. VINKEN Niedersächsisches Landesamt für Bodenforschung Hannover

Als Ex-officio-Mitglieder zählen ferner:

L.-K. Königsson (Präsident der INQUA Holocene Commission)

Uppsala Universitet, Kvartärgeologiska avdelningen

Uppsala/Schweden und

D. J. Colquhoun (Präsident der INQUA-Shorelines Commission)

Department of Geology, University of South Carolina

Columbia, South Carolina, U.S.A.

$$
\text { Anlage } 2
$$

Küstenforscher aus dem Bereich der Bundesrepublik Deutschland, die als offizielle Kontaktpersonen zu bzw. als Mitarbeiter in internationalen Organisationseinheiten tätig sind:

Prof. Dr. K.-E. BeHre - mailing list of INQUA-subcommission on Shorelines of Northwestern Europe. - Niedersächsisches Landesinstitut für Marschen- und Wurtenforschung, Viktoriastraße 26/28, D-2940 Wilhelmshaven

Prof. Dr. H. KLUG - corresponding member der INQUA-subcommission on Shorelines of Northwestern Europe. - Geographisches Institut der Universität Regensburg, Universitätsstraße 31, D-8400 Regensburg

Prof. Dr. R. Köster - corresponding member der INQUA-subcommission on Shorelines of Northwestern Europe. - Geol.-Paläontologisches Institut der Universität Kiel, Olshausenstraße 40-60, D-2300 Kiel

Dr. H. STREIF

- full member der INQUA-subcommission on Shorelines of Northwestern Europe. - Niedersächsisches Landesamt für Bodenforschung, Stilleweg 2, D-3000 Hannover 51

Dr. R. VINKEN

- Mitglied des Executive Board und nationaler Projektleiter des IGCP Projects No. 61. - Niedersächsisches Landesamt für Bodenforschung, Stilleweg 2, D-3000 Hannover 51. 